\title{
ORIGINAL ARTICLE Olfactory stem cells reveal MOCOS as a new player in autism spectrum disorders
}

\author{
F Féron ${ }^{1,2,10}$, B Gepner ${ }^{2,10}$, E Lacassagne², D Stephan ${ }^{2}$, B Mesnage $^{2}$, M-P Blanchard ${ }^{3}$, N Boulanger $^{4}$, C Tardif $^{5}$, A Devèze $^{6,7}$, \\ S Rousseau ${ }^{8}$, K Suzuki $^{9}$, JC Izpisua Belmonte $^{9}$, M Khrestchatisky $^{2}$, E Nivet $^{2}$ and M Erard-Garcia ${ }^{2}$
}

With an onset under the age of 3 years, autism spectrum disorders (ASDs) are now understood as diseases arising from pre- and/or early postnatal brain developmental anomalies and/or early brain insults. To unveil the molecular mechanisms taking place during the misshaping of the developing brain, we chose to study cells that are representative of the very early stages of ontogenesis, namely stem cells. Here we report on MOlybdenum COfactor Sulfurase (MOCOS), an enzyme involved in purine metabolism, as a newly identified player in ASD. We found in adult nasal olfactory stem cells of 11 adults with ASD that MOCOS is downregulated in most of them when compared with 11 age- and gender-matched control adults without any neuropsychiatric disorders. Genetic approaches using in vivo and in vitro engineered models converge to indicate that altered expression of MOCOS results in neurotransmission and synaptic defects. Furthermore, we found that MOCOS misexpression induces increased oxidative-stress sensitivity. Our results demonstrate that altered MOCOS expression is likely to have an impact on neurodevelopment and neurotransmission, and may explain comorbid conditions, including gastrointestinal disorders. We anticipate our discovery to be a fresh starting point for the study on the roles of MOCOS in brain development and its functional implications in ASD clinical symptoms. Moreover, our study suggests the possible development of new diagnostic tests based on MOCOS expression, and paves the way for drug screening targeting MOCOS and/or the purine metabolism to ultimately develop novel treatments in ASD.

Molecular Psychiatry (2016) 21, 1215-1224; doi:10.1038/mp.2015.106; published online 4 August 2015

\section{INTRODUCTION}

Autism spectrum disorders (ASDs) are complex neurodevelopmental diseases arising from multifactorial genetic, epigenetic and environmental origins. ${ }^{1-4}$ Systems-level connectivity features ${ }^{5}$ and plausible neuroanatomical, ${ }^{6}$ cellular $^{7,8}$ and molecular ${ }^{9}$ underpinnings of ASD have been highlighted. Although hundreds of susceptibility genes have been identified, being generally involved in neurobiological functions such as neurogenesis, synaptogenesis and neurotransmission, ${ }^{10}$ collectively they account for $10-20 \%$ of ASD cases at most. ${ }^{11}$ Accordingly, discovering common genetic traits being more representative of ASD population becomes a priority for early diagnoses, ${ }^{12}$ investigation of ASD physiopathology and identification of new therapeutic targets.

ASD-associated profiles are underpinned by atypical neural development often accompanied by epilepsy, gastrointestinal disorders and other comorbid disorders. ${ }^{3}$ Despite numerous studies, the etiopathology as well as the physiopathogeny of these disorders remain largely elusive. To date, genetic studies on ASD have mainly used cellular material that, even though being sufficient for mutagenic studies, might be irrelevant to identify gene misexpression during development. To seek for novel candidates having a role in ASD, we chose to study cells that are representative of early stages of ontogenesis. To this end, we decided to use human nasal olfactory stem cells (OSCs) displaying multipotent properties ${ }^{13,14}$ and proved to be useful for transcriptomic analyses in the context of brain disorders. ${ }^{15,16}$
Our study is based on a relatively homogeneous cohort of nine adults with severe autism and low to very low developmental disabilities plus two adults with mild or moderate autism and no or mild cognitive abilities (Asperger syndrome or high-functioning autism), paired with 11 age- and gender-matched control individuals. We first chose a non-hypothesis-driven approach and looked for transcriptome anomalies, using pangenomic cDNA microarrays. We observed a dysregulated expression of genes already associated to ASD. Of note, for the very first time, we also identified a new candidate gene, the MOlybdenum COfactor Sulfurase (MOCOS). MOCOS is known as an enzyme of the purine metabolism that sulfurates the molybdenum cofactor, thus allowing the two downstream enzymes-xanthine dehydrogenase $(\mathrm{XDH})$ and aldehyde oxidase (AOX1) - to be active. ${ }^{17}$ Considering the role of $\mathrm{XDH}$ and $\mathrm{AOX}$ in purine degradation as well as in the Redox balance, and so in cellular stress response, the role of MOCOS appears to be important though poorly investigated yet. MOCOS mutations have been associated with xanthinuria type II, a disorder characterized by an abundant excretion of urinary xanthine and a diminished production of uric acid, in both serum and urine. ${ }^{18-20}$ To date, however, nothing is known about a putative role of this gene on cerebral functions. Therefore, and considering the clinical symptoms associated to ASD, our data led us to study whether a dysregulation of MOCOS expression could have an impact on functions known to be involved in the related disease phenotype.

\footnotetext{
${ }^{1}$ Inserm CBT 1409, Centre d'Investigations Cliniques en Biothérapie, Marseille, France; ${ }^{2}$ Aix Marseille Université, CNRS, NICN UMR 7259, Marseille, France; ${ }^{3}$ Aix Marseille Université, CNRS, CRN2M UMR 6231, Marseille, France; ${ }^{4}$ Aix Marseille Université, TAGC UMR 1090, Marseille, France; ${ }^{5}$ Aix Marseille Université, PsyCLE, EA 3273, Aix en Provence, France; ${ }^{6}$ AP-HM, Département ORL, Marseille, France; ${ }^{7}$ Aix Marseille Université IFSTTAR, UMRT 24, Marseille, France; ${ }^{8}$ AP-HM, Département Anesthésie, Marseille, France and ${ }^{9}$ Gene Expression Laboratory, The Salk Institute for Biological Studies, La Jolla, CA, USA. Correspondence: Professor F Feron or Dr M Erard-Garcia, Faculty of Medicine, Aix Marseille Université, CNRS, NICN UMR 7259, 13344 Marseille, France.

E-mail: francois.feron@univ-amu.fr or madeleine.garcia@univ-amu.fr

${ }^{10}$ These authors contributed equally to this work.

Received 28 January 2015; revised 6 May 2015; accepted 15 June 2015; published online 4 August 2015
} 
We found that the genetic ablation of mocs-1 (the MOCOS ortholog) in Caenorhabditis elegans (C. elegans) induces an alteration of the response to oxidative stress and is responsible for abnormal neurotransmission phenotypes. Then, we confirmed these data in human cells. First, in OSCs that displayed a higher production of reactive oxygen species (ROS). Second, in human induced pluripotent cells (iPSCs) in which the gene MOCOS was partially knocked down and showed a reduced synaptogenesis when differentiated into neurons.

\section{MATERIALS AND METHODS}

\section{Participants}

A complete written and oral information on the goal and procedure of this research was provided to the participants or their legal tutors and a signed informed consent was obtained from all of them, before their involvement in the study.

All procedures were approved by the local ethical committee (Comité de Protection des Personnes, files \#205016 and \#205017) of Marseille. Patients with ASD $(n=11)$ were recruited in three public hospital units (Valvert Hospital, Marseille; Montperrin Hospital, Aix-en-Provence and Edouard Toulouse Hospital, Marseille), in two socio-medical private units (Foyer d'accueil médicalisé, Pélissanne and Service d'accompagnement medico-social pour adultes autistes, Salon de Provence) and through a specialized ASD diagnosis consultation (Montperrin Hospital, Aix-enProvence). The patients were diagnosed according to ICD-10 (World Health Organization, 1993) and DSM-5 (American Psychiatric Association, 2013) criteria for pervasive developmental disorders and ASDs, respectively. Control patients were recruited to match the age and gender of each ASD patient that was endorsed in the cohort. The healthy controls were neither presenting a neuropsychiatric disorder nor taking medication. Demographics of the cohort are summarized in Supplementary Tables 1 and 2. Details on risk factors, comorbidity, medication and magnetic resonance imaging data of ASD patients of the cohort are given in Supplementary Tables 3.

\section{Nasal biopsies}

Nasal biopsies were performed by an ENT surgeon as we previously described. ${ }^{21}$ For ASD patients, the tissue was biopsied right after a brain magnetic resonance imaging examination, requiring a general anesthesia in all of them. Control participants were biopsied during an ENT surgery.

\begin{abstract}
Animals
C. elegans strains were maintained at $20^{\circ} \mathrm{C}$ under standard growth conditions. Strains used in these studies include: N2 (wild type), mocs-1 (tm3708), mocs-1(ok3439), F15E6.6 (ok1816), ric-3(md158), eri-1(mg366), mev-1 (kn1), arls37 [Pmyo-3::ssGFP], nuls183 [unc-29-nlp-21::YFP] ${ }^{22}$ and VAP1::RFP (generous gift of L Bianchi, University of Miami). The modified WRM0612B_E02 fosmid was obtained from the TransgeneOme project. In this vector, the green fluorescent protein (GFP) coding sequence was fused to the $3^{\prime}$ end of mocs- $1 .{ }^{23}$ Wild-type transgenic animals were selected after bombardment of unc-119(ed3) mutants. The tm3708 mutant contains a 621bp deletion that removes intron 1 and part of exon 2 (see Figure 3a). The tm3708 deletion was predicted to represent a null allele because the mutation was generating a premature amber stop codon after only $10 \%$ of the coding region. The ok3439 deletion removes part of exon 2 and all subsequent exons up to exon 5 (see Figure 3a). This mutant displays an in-
\end{abstract}

frame deletion of the L-cysteine desulfurase domain: ok3439 is predicted to be devoid of 163 amino acids in a region known to bind the PLP cofactor and mobilize sulfur from L-cysteine. Thus, ok3439 deletion mutants are supposed to be free of their cysteine desulfurase activity, whereas the MOSC domain remains intact. The ok1816 mutant is a deletion mutant for F15E6.6.

C57/Bl6 mice (8-week old) were used to evaluate the expression of MOCOS in various organs as well as to establish primary cultures of neurons. All murine experiments were conducted according to the guidelines of the Ethics Committee of the Medical Faculty of Marseille and conform to National and European regulations (EU directive Nu 86/609). Animals were housed in air-flow racks on a restricted access area and maintained on a 12$\mathrm{h}$ light/dark cycle at a constant temperature $\left(22 \pm 1{ }^{\circ} \mathrm{C}\right)$. For animal studies, no method of randomization was used and no blinding was done.

\section{Cell culture}

All cell culture-related works were performed as described in Supplementary Information Methods.

\section{Microarray gene expression analysis}

OSCs from 22 individuals were collected and processed for hybridization on pangenomic CDNA arrays and microarray data analysis as described in Supplementary Information Methods. Microarray data have been deposited in NCBI-GEO under the accession number GSE63524.

\section{RNA isolation and real-time PCR analysis}

RNA samples extracted from OSCs were tested with qRT-PCR as described in Supplementary Information Methods.

\section{Western blot analysis}

Protein expression analyses in OSCs were performed using a standard protocol as described in Supplementary Information Methods.

\section{Paraquat assay}

For paraquat sensitivity during development, adult animals were placed on plates containing increasing concentrations of paraquat $(0.15,0.22,0.44$, 0.66 and $4 \mathrm{~mm}$ ), allowed to lay eggs for $6 \mathrm{~h}$ and then removed. Over a 4-day period, the proportion of animals at every development stage (L1-L4) progeny was scored. The total number of worms that survived after exposure to $4 \mathrm{~mm}$ of paraquat was assessed. To test the sensitivity of adult worms to oxidative stress, young adults (day 1) were transferred to fresh plates containing $4 \mathrm{~mm}$ of paraquat. The total number of worms that survived was counted after 24,48 and $72 \mathrm{~h}$.

\section{RNAi procedures}

Bacteria expressing dsRNA directed against R03A10.3, F55B11.1 and L4440 were part of a C. elegans RNAi library expressed in Escherichia coli (Geneservice, Cambridge, UK). RNAi was delivered by feeding as previously described. ${ }^{24}$ In brief, L4 hermaphrodites were placed on RNAi feeding plates. After 4 days, F1 progeny were transferred either to the plates filled with nematode growth medium agar supplemented with $20 \mathrm{~mm}$ paraquat or to control unsupplemented nematode growth medium plates.

\section{ROS assay in OSCs}

Generation of intracellular ROS in human OSCS was measured by staining with CellROX Green Reagent (\#C10422, Life Technologies, Carlsbad, CA, USA)

Figure 1. Human nasal olfactory stem cells (OSCs) reveal a subtle but robust downregulation of MOCOS in ASD patients. (a) Network analysis of differentially expressed genes, in at least four ASD patients of the cohort, using ingenuity pathways analysis (IPA). Note that TGFB1/WNT catenin are the most affected signaling pathways. Solid and dashed lines indicate direct and indirect interactions, respectively. Consistently overexpressed or underexpressed genes are in red or blue boxes, respectively. Up and down misexpressed genes are in green boxes. (b) Volcano plot distribution of the microarray data. The blue and red vertical bars indicate magnitude fold changes (FC) over [1.5] and the dashed black line shows the $P=0.05$ threshold, with points above the line having $P<0.05$. Note that the average for all MOCOS probes (10 in total on the microarray) display the same pattern of expression. (c) Graph depicting MOCOS gene expression FC in ASD patients, relative to control patients, as detected by microarray and RT-qPCR. Note that highly similar results were obtained with both the techniques. (d) Representative pictures showing MOCOS immunostaining of nasal OSCs isolated from one ASD patient (right panel) and his matched healthy individual (left panel). Nuclei were counterstained with DAPI. (e) Representative western blot for the detection of MOCOS and GAPDH expression in control and ASD patients. (f) Quantification of MOCOS expression, expressed as a ratio to GAPDH, in ASD and control OSCs using the western blot technique. Data are represented as mean \pm s.d. ${ }^{*} P<0.05$. Scale bars: $10 \mu \mathrm{m}$ (d). 
a
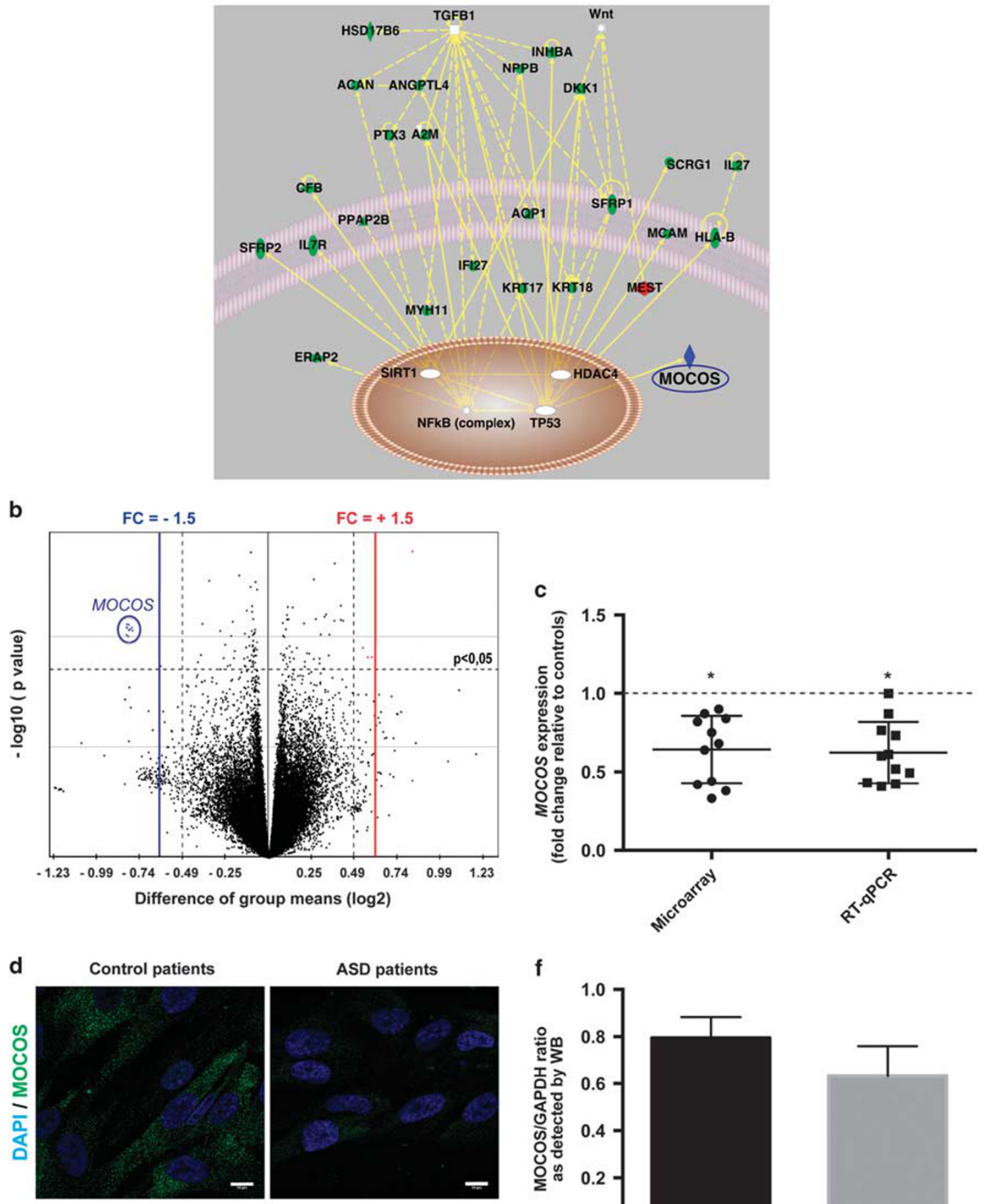

e

Control patients

ASD patients
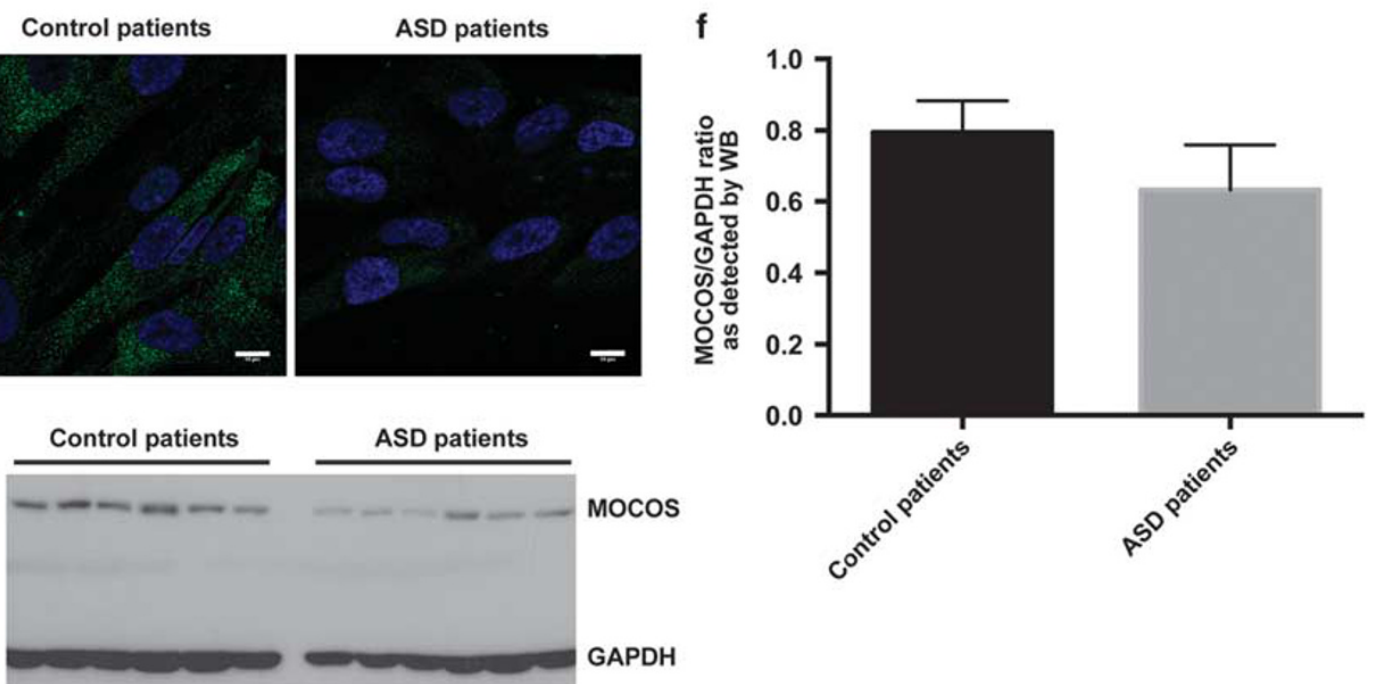

GAPDH 
according to the manufacturer's instructions as described in Supplementary Information Methods.

Aldicarb and levamisole-induced paralysis assays

Acute sensitivities to aldicarb and levamisole were assayed in paralysis assays as previously described. ${ }^{25}$ For the aldicarb assay, 30-40 young adult animals were transferred to nematode growth medium agar plates containing $0.25 \mathrm{~mm}$ aldicarb and day 1 adult worms were scored every $30 \mathrm{~min}$ (moving ratio). For the levamisole-induced paralysis assay, nematode growth medium agar plates containing various concentrations of levamisole $(0.1,0.2,0.5,0.8$ and $1 \mathrm{~mm}$ ) were prepared 1 day before the assay and adult worms were tested for complete paralysis after $60 \mathrm{~min}$ of drug exposure. Animals were considered paralyzed if they failed to move when prodded with a platinum wire.

Fluorescence imaging and quantitative analysis of NLP-21 and GFP-containing vesicles

Analysis of NLP-21 and GFP-containing vesicles was performed in live animals as described in Supplementary Information Methods.

Generation of the MOCOS gene-knockout human iPSCs

Gene-edited human iPSCs were generated using the TALENs technology as described in Supplementary Information Methods.

Neuronal differentiation from human iPSCs

First we derived neural stem cells from iPSCs $\left(\mathrm{MOCOS}^{+/+}\right.$and $\left.\mathrm{MOCOS}^{+/-}\right)$as described elsewhere ${ }^{26}$ with slight modifications as described in Supplementary Information Methods.

\section{Immunochemistry}

Immunostainings were performed using a standard protocol as described in Supplementary Information Methods.

\section{Statistical evaluation \\ Statistical analyses were performed by using standard unpaired Student's t- tests (two-tailed, $95 \%$ confidence intervals) or one-way analysis of variance, and were carried out using Prism software (GraphPad Software, La Jolla, CA, USA) or Excel software (Microsoft, Redmond, WA, USA). For microarray data analyses, right-tailed Fisher's exact test was used to calculate a $P$-value determining the top statistically significant biological functions assigned to the data set. Normal distribution was confirmed before applying statistical tests. All data are presented as mean \pm s.d. and represent a minimum of three independent experiments with at least three technical replicates except when otherwise indicated. Estimate of variance was performed within each group of data being statistically compared. A value of $P<0.05$ was considered significant.}

\section{RESULTS}

OSCs reveal changes in MOCOS expression in ASD patients

We first established the transcriptome profiles of OSCs isolated from nasal biopsies ${ }^{21}$ of 11 patients diagnosed with ASD and 11 age- and gender-matched control individuals (Supplementary Tables 1). Gene microarray analysis identified 156 genes that were differentially expressed in at least one ASD patient (Supplementary Table 6), of which 31 were dysregulated in more than a third of the cohort (Supplementary Table 6, in red). Notably, 9 out of these 156
genes-ADAM23, CADM1, FOS, FOSB, JAG1, MEST, OXTR, SFRP1 and XIST-have been previously associated with ASD. ${ }^{27}$ Ingenuity pathway analysis on the pool of differentially regulated genes identified developmental disorders and gastrointestinal diseases as two of the most represented categories associated with these genes (Supplementary Table 7). Furthermore, ingenuity pathway predictive analysis highlighted that, among the main upstream regulators of the differentially expressed genes, three-TNF, IFNG and ILIB-are associated with inflammation (Supplementary Table 7), a functional process widely associated with ASD. ${ }^{28,29}$ In addition, gene cluster analysis revealed that all patients were affected by a partial dysregulation of the TGF- $\beta /$ Wnt pathways (Figure 1a and Supplementary Table 8), known to have an important role in ASD. $^{30,31}$ Second in line among the most affected pathways, purine metabolism appeared largely disturbed with the misexpression of at least one of the two enzyme-coding genes, MOCOS and AOX1, in 10 out of 11 patients (Supplementary Table 8). Although AOX1 was found differentially expressed in 3 out of 11 patients, its pattern of expression was heterogeneous when compared with the subtle but robust MOCOS downregulation displayed by $\sim 80 \%(8 / 11)$ of the patients composing our cohort (Figures $1 \mathrm{~b}$ and c). Importantly, MOCOS downregulation in ASD patients' OSCs was confirmed at both the RNA and protein levels (Figures 1c-f). Interestingly, metabolic disorders of purine metabolism have been described to affect the nervous system and able to induce autistic features. ${ }^{32,33}$ Accordingly, we decided to focus on MOCOS, an enzyme acting upstream in the purine catabolism pathway, and investigated its putative role in ASD.

The fact that MOCOS adds sulfur on the molybdenum cofactor and turn $\mathrm{XDH}$ into its active form to catalyze the oxidation of hypoxanthine to xanthine and, further down, the oxidation of xanthine to uric acid, led us to retrospectively investigate whether our patients suffered from xanthinuria. ${ }^{17,20}$ In addition, and because creatinine and ferritin are often associated with uric acid production, we also assessed their expression in the blood and urine. Except in one patient that displayed a high level of xanthine, our analyses did not reveal any direct correlation between these three measurements (Supplementary Table 9). These results led us to propose that MOCOS misexpression might affect other functions and we decided to investigate whether it could have a role in brain functions.

\section{Strong MOCOS expression in the brain and other tissues}

We first sought to look at the dynamic expression of MOCOS in the brain. Using the Allen Brain Atlas tool, we found that MOCOS is expressed in numerous areas of the developing and adult human brain. Confirming the expression pattern suspected by transcriptomic analyses in human, adult mouse brain tissue analysis confirmed the presence of MOCOS, at the protein level, in multiple brain areas such as the hippocampus, the cortex, the cerebellum and the brainstem (Figure $2 a$ and Supplementary Figure 1a). Furthermore, MOCOS was readily detected in MAP2+ murine neurons in vitro and in vivo (Figures $2 \mathrm{~b}-\mathrm{d}$ and Supplementary Figure 1a). In parallel, we found that MOCOS is expressed in other tissues such as the intestine, the kidney and the liver (Figures 2e

Figure 2. MOCOS is expressed in various C. elegans and mouse organs including the brain. (a) Sagittal section of an adult mouse brain showing the very high expression of MOCOS in the cortex (CTX), the hippocampus (HIP), the outer layers of the cerebellum (CB) and the dorsal area of the olfactory bulb (OB). (b) Representative picture of MAP2+ neurons (red) in the CA1 layer of the hippocampus (dashed white box in a) immunostained for MOCOS (green) and counterstained with DAPI. On the right panel, high magnification view. (c and d) Representative pictures at low (c) and higher magnification (d) of cultivated murine neurons immunostained with the indicated antibodies. (e and $\mathbf{f}$ ) Mouse gut sections displaying an intense MOCOS staining in ciliated cells (e) and glands (f). (g) MOCS1 expression in the developing C. elegans was monitored using a mocs-1::GFP transgenic strain. Representative pictures showing that MOCS1 was found highly expressed in the intestine and the head in the embryo (left panel at the gastrula stage, DIC: differential interference contrast), at larval stages (L1 on the picture) and maintained throughout the adult life. Arrows indicate sheath (white) and socket (yellow) glial-like cells, as well as intestinal (red) cells. Scale bars: $100 \mu \mathrm{m}$ (e and f, left panels), $20 \mu \mathrm{m}$ (g, middle panels), $10 \mu \mathrm{m}$ (b, c, d; e, f, right panels; $\mathbf{g}$, left panels and right panel). 
and $f$ and Supplementary Figure $1 b$ ). As MOCOS is a gene highly conserved across species, we additionally analyzed its expression in C. elegans by using a transgenic strain in which the GFP coding sequence was fused to the $3^{\prime}$ end of the orthologous gene mocs-1. ${ }^{23}$ We observed GFP expression as early as the eight-cell stage of the intestine and throughout the larval and adult life of the animal in the cytoplasm of cells in the intestine as well as in the head (Figure $2 \mathrm{~g}$ ). Regarding the latter, mocs-1 reporter expression was detected in sheath- and socket-glial cells (Figure $2 \mathrm{~g}$ and Supplementary Figure 1c). Altogether, these data suggest that throughout evolution, MOCOS has an important role in development and adult life. Importantly, the multicellular/multiorgan
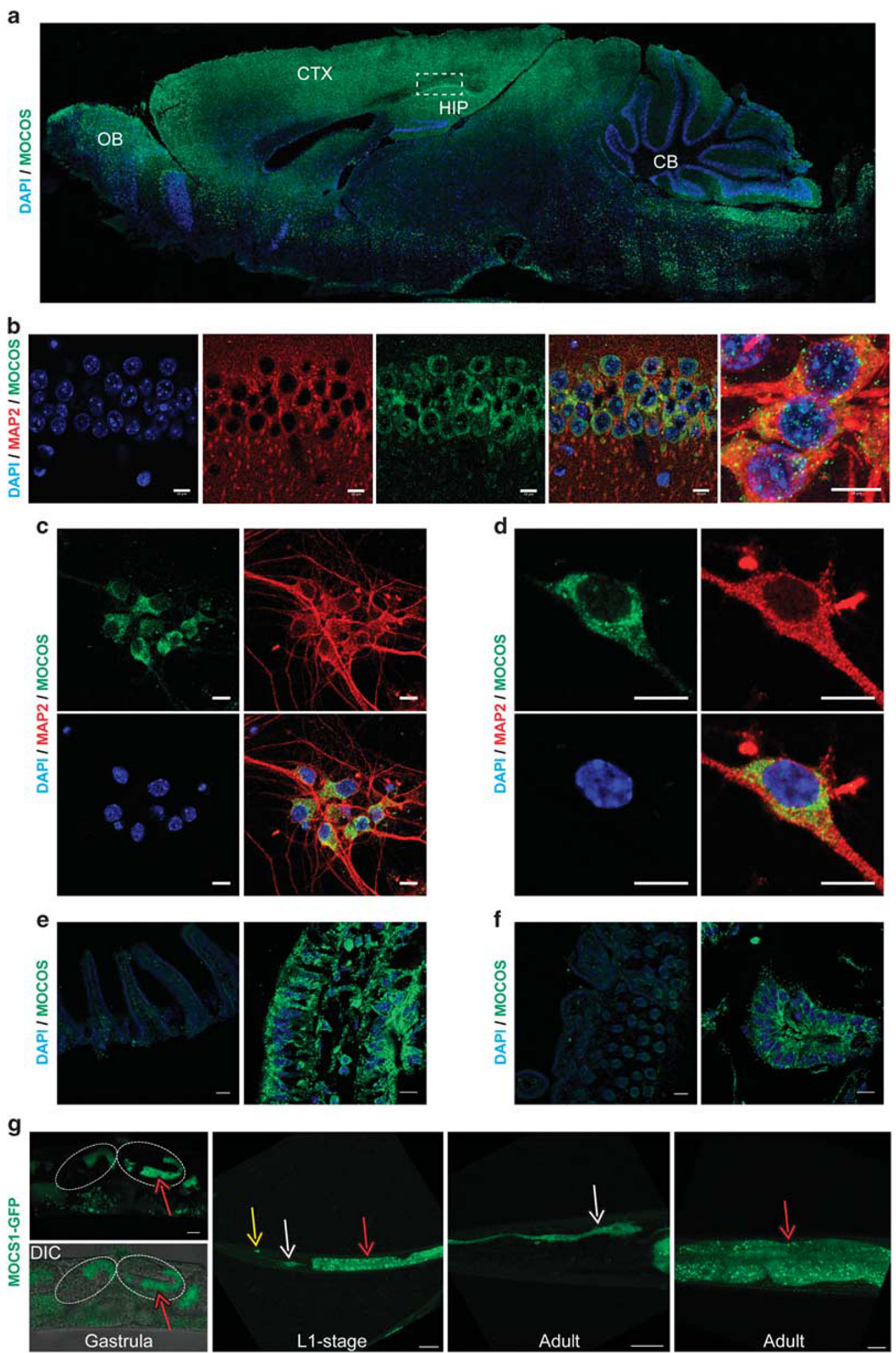
distribution of MOCOS fits with the search of a pleiotropic gene that could explain complex disorders such as ASD.

\section{MOCOS misexpression increases sensitivity to oxidative stress}

To investigate whether altered expression of MOCOS correlates with ASD-associated phenotypes, we used two independent $C$. elegans strains mutant for mocs-1 and bearing a nonsense mutation (tm3708) or a frameshift mutation (ok3439) (Figure 3a). Several studies having reported a greater oxidative stress in children with autism, ${ }^{34,35}$ we first assessed the sensitivity of mocs-1 mutants to paraquat, an oxygen radical-generating agent. Although development was slowed down in all worms exposed to $0.22 \mathrm{~mm}$ of paraquat, $82 \%$ and $52 \%$ of tm 3708 and ok3439 mutants, respectively, reached the L4 larval stage when compared with wild-type animals (Figure 3b), demonstrating an increased sensitivity to oxidative stress. Interestingly, the observed effects were dose dependent as larval development was blocked at the L2/L3 stages in mocs-1 mutants treated with higher concentrations $(\geqslant 0.44 \mathrm{~mm})$, and led to a significantly lower survival rate in tm3708 (12\%) and ok3439 (0\%) mutants comparatively to wildtype $(98.5 \%)$ animals exposed at $4 \mathrm{~mm}$ of paraquat (Figures $3 \mathrm{~b}$ and c). Confirming the role and specificity of mocs- 1 in response to paraquat, similar results were obtained with eri-1 animals fed with an RNAi against R03A10.3, the mocs-1 predicted gene (Figure $3 \mathrm{~d}$ ). Similarly, we observed that exposure to paraquat at an adult stage strongly decreases the viability of mocs-1 mutants as well as eri-1 animals fed with R03A10.3 RNAi when compared with their respective controls (Figures $3 e$ and $f$ ). Because the sulfuration of the molybdenum cofactor is essential for the final maturation of xanthine oxydoreductase and AOX1 enzymes, we therefore evaluated oxidative-stress sensitivity during the development of worms expressing reduced levels of these two molybdoflavoenzymes. In C. elegans, F55B11.1 is similar in sequence to human $\mathrm{XDH}$ (47\% sequence identity), whereas F15E6.6 is predicted to encode one homologue of $A O X 1 .^{36}$ Interestingly, F15E6.6 (ok1816) mutant worms and eri-1 animals fed with F55B11.1 RNAi exhibited an increased and similar sensitivity to paraquat (Supplementary Figure 2). Furthermore, sensitivity to oxidative stress was increased when both genes were depleted simultaneously (Supplementary Figure 2).

Then, we evaluated whether a similar feature could be observed in patients' OSCs displaying MOCOS downregulation. Validating further our initial observations in worms, those cells revealed a higher production of ROS, at baseline and $1 \mathrm{~h}$ after staurosporine treatment, when compared with their respective controls (Figure $3 \mathrm{~g}$ ). Notably, this feature is in line with the fact that MOCOS adds a sulfur atom on the molybdenum cofactor of AOX1, an enzyme involved in the regulation of ROS homeostasis. Altogether, these data demonstrate that altered expression of MOCOS may increase sensitivity to oxidative stress, a feature that has been widely reported to contribute to the development and clinical manifestations of autism. ${ }^{34,35}$ Moreover, our data indicate that increased sensitivity to oxidative stress may reflect, at least partly, impaired XDH and AOX1 function.

\section{MOCOS has a role in neurotransmission}

To date, the physiopathology of ASD is commonly accepted to be partly due to a defective regulation of neurotransmission., ${ }^{1,37}$ Accordingly, we assessed synaptic function in mocs-1 mutants. To this end, we evaluated the synaptic transmission at the neuromuscular junction by monitoring the sensitivity of mocs- 1 mutants to the paralyzing effects of aldicarb, an acetylcholinesterase inhibitor. ${ }^{25}$ We found that $\operatorname{tm} 3708$ and ok3439 animals were resistant to aldicarb when compared with N2 wild-type worms (Figure 4a). Notably, we observed similar paralytic and lethal effects in wild-type and mocs-1 mutant worms exposed to increasing concentrations of levamisole, ruling out an alteration of the postsynaptic receptor function (Supplementary Figure 3a). Next, we reasoned that aldicarb resistance in mocs-1 mutants could be the consequence of a decreased neuropeptide secretion. Accordingly, we analyzed the secretion of the YFP-tagged neuropeptide NLP-21 in cholinergic motor neurons. As shown in Figure 4, NLP-21 puncta fluorescence intensity was significantly reduced in the dorsal cord axons of $t m 3708$ hypomorph mutants $(23.5 \pm 3 \%)$ when compared with wild-type animals and a similar decrease was observed in coelomocytes (29.2 $\pm 8.5 \%$ ) of both groups (Figures $4 \mathrm{~b}$ and c). Together, these data suggested a reduced number of dense core vesicles in the axons as well as a quantitatively reduced but functionally normal neuropeptide secretion. Further investigations on tm3708 mutants showed a significant accumulation of NLP-21 in the soma of dorsal cord neurons and demonstrated that NLP-21 was localized into larger vesicles $\left(\geqslant 0.2 \mu \mathrm{m}^{2}\right)$ when compared with control animals, suggesting that defects in neuropeptide packaging account for the observed phenotype (Figures $4 d-f$ ). Of note, the observed phenotypes were confirmed in tm3708 mutants harboring an integrated pmyo-3::ssGFP transgene (Supplementary Figures 3b-d). Interestingly, similar analyses on ok3439 neomorph mutants revealed no difference of NLP-21 distribution in dorsal cord neurons but a significant decrease in number within coelomocytes (Figures 4b-e), highlighting that ok3439 mutants have decreased neuropeptide secretion when compared with wildtype animals. In summary, although the two mocs-1 mutant strains were found differentially affected, probably due to the nature of the mutation, our results indicate that deregulation of MOCS1 expression may affect the neuropeptide secretory system.

This finding prompted us to use the iPSC technology for validating the role of MOCOS in human neuronal cells. Using TALEN technology, we successfully derived four heterozygous knockout $\left(\mathrm{MOCOS}^{+/-}\right.$) iPSCs lines (Figure $4 \mathrm{~g}$ and Supplementary Figure 4). Notably, several attempts at establishing homozygous knockout $\left(\mathrm{MOCOS}^{-1-}\right)$ iPSC lines failed, suggesting an important role of MOCOS in human cell homeostasis, at least during early development. To investigate whether MOCOS can have a role in neurotransmission, MOCOS $^{+/-}$iPSCs and their respective isogenic

\footnotetext{
Figure 3. MOCOS expression alterations induce increased stress sensitivity. (a) Cartoon depicting the main structural characteristics of the gene encoding the molybdenum cofactor sulfurase in plants (A-thaliana), humans and $C$. elegans. Note the sequence homology between the latter two. The nonsense tm 3708 mutant lacks intron 1 and part of exon 2, whereas the frameshift ok3439 mutant lacks part of exon 2 and all subsequent exons up to exon 5. (b) Larval development is slowed down, in a dose-dependent manner, in mocs-1 mutants exposed to paraquat. N2 animals were used as wild-type controls. mev-1 worms, a strain hypersensitive to paraquat, was used as an experimental control for the paraquat assay. (c) Bar chart depicting the reduced survival rate of mocs-1 larval mutants exposed to high level of paraquat (4 mm). (d) Disturbed larval development in eri-1 animals fed with an RNAi directed against mocs-1 (R03A10.3) when compared with control eri-1 worms fed with RNAi against the empty vector L4440. (e) Time course survival rate of mocs-1 adult mutants exposed to high level of paraquat (4 mm). $\mathrm{N} 2$ and mev-1 animals were used as controls as defined in b). (f) Bar chart demonstrating the reduced survival rate of eri-1 adult animals fed with an RNAi directed against mocs-1 (R03A10.3) comparatively to their control (fed with RNAi against L4440), after exposure to high level of paraquat $(4 \mathrm{~mm})$. (g) ASD patients' nasal OSCs produce higher levels of ROS, at baseline and $1 \mathrm{~h}$ after staurosporine treatment. Data are represented as mean \pm s.d. ${ }^{*} P<0.05 . n=80-100$ animals per group. Experiments were run in triplicate.
} 
$\mathrm{MOCOS}^{+/+}$control lines were differentiated into neurons (Figure 4h). The synaptic compartment being an important element for neurotransmission, we analyzed the number of synaptic buttons in iPSCs-derived neurons (Figure 4h). Of utmost importance, and even though we did not observe difference in their capacity to generate TUJ1-expressing neurons, those derived from $\mathrm{MOCOS}^{+/-}$

a
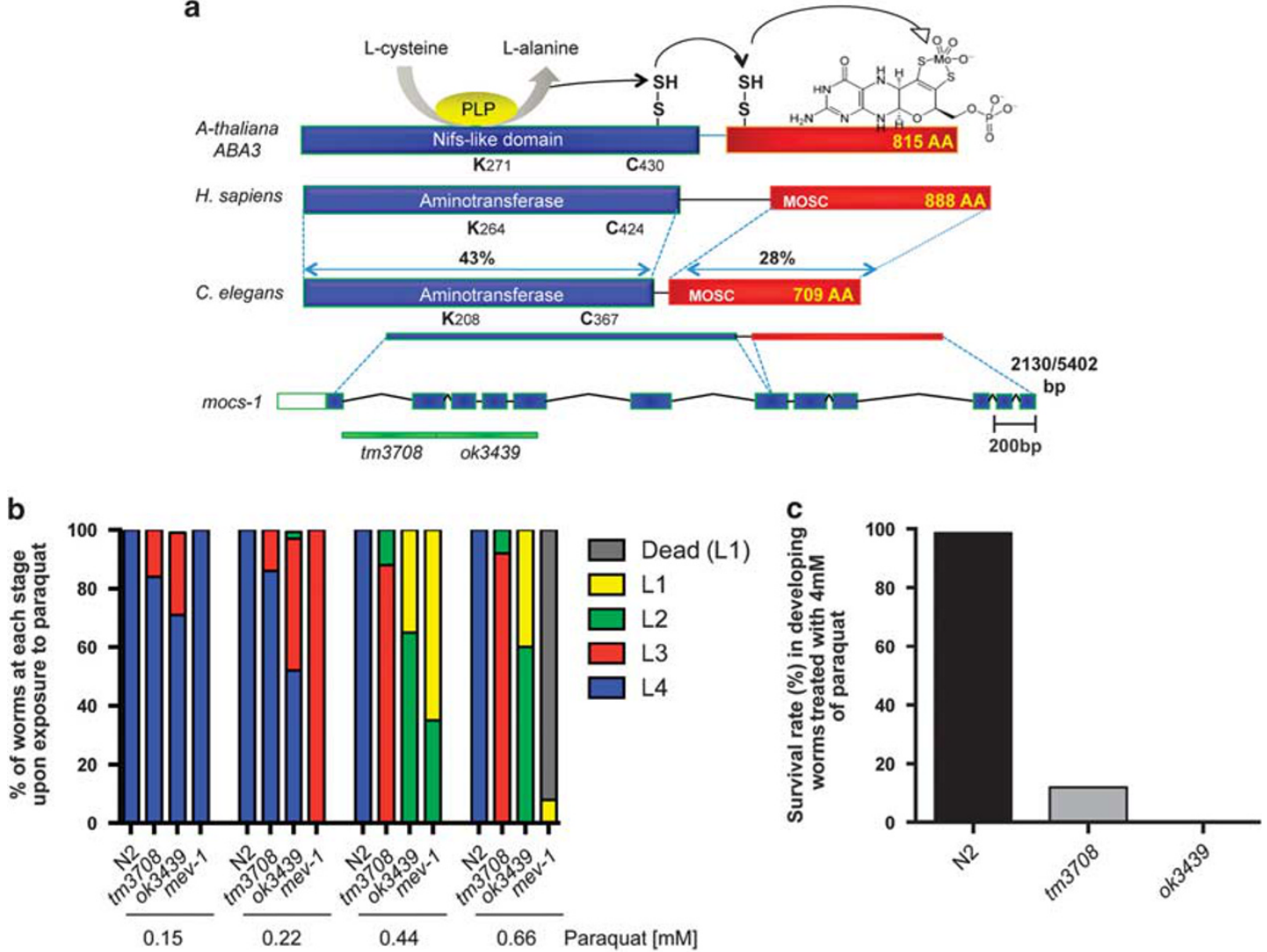

d
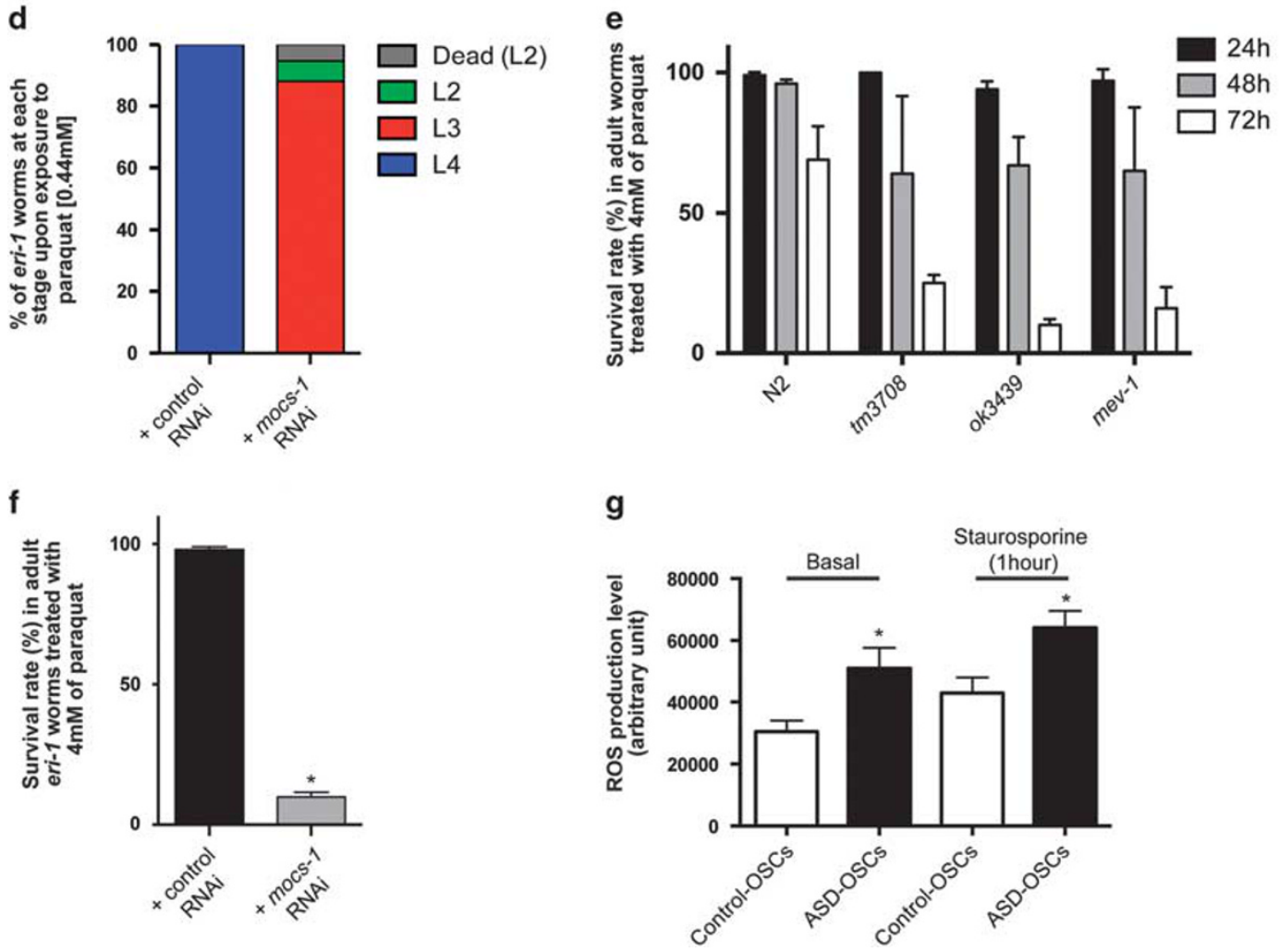
a

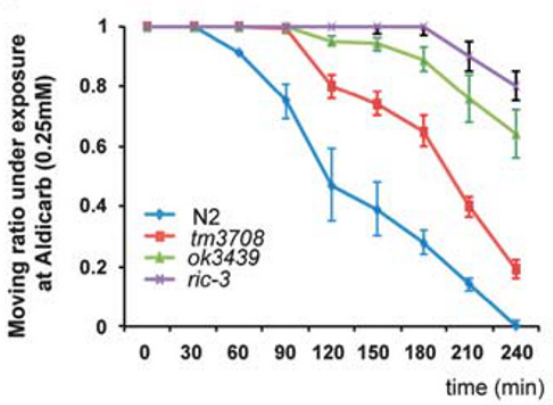

b

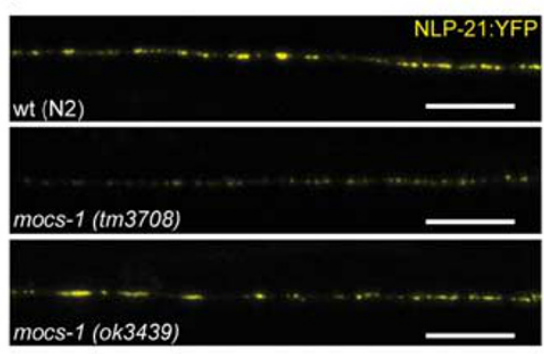

mocs-1 (ok3439)

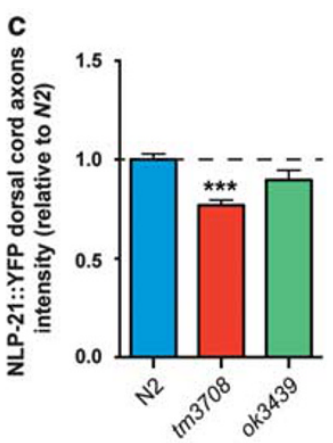

f
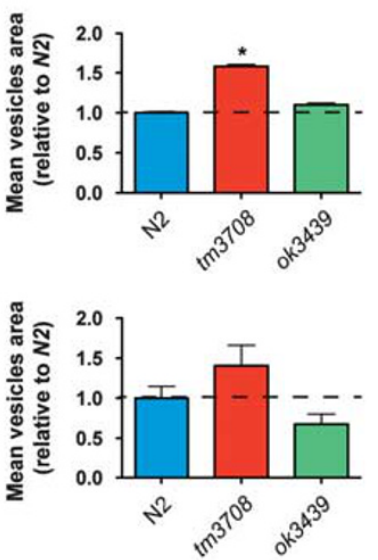

d

wt (N2)
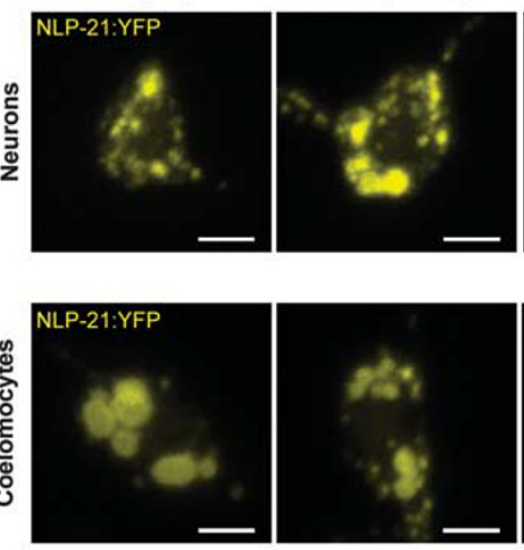

e
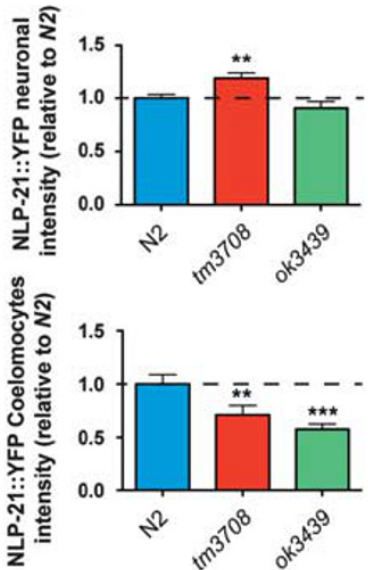
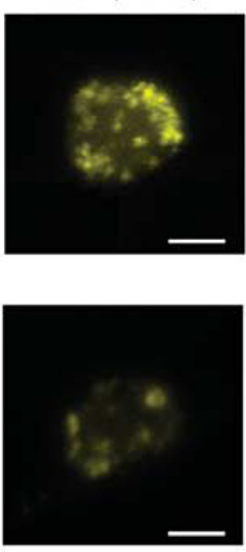

\section{g}

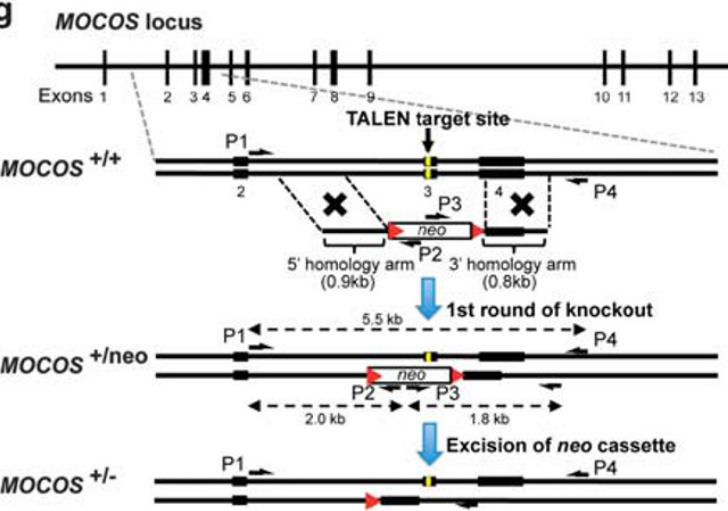

h

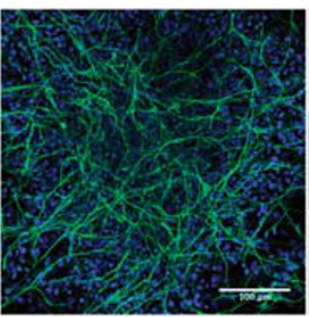

TUJ-1 / DAPI

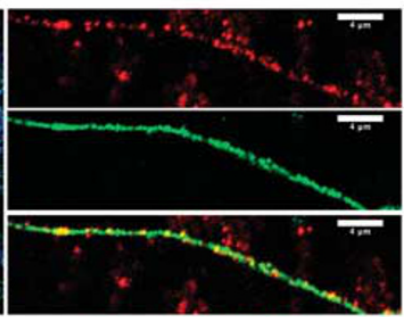

TUJ-1/ SYNAPSIN

i

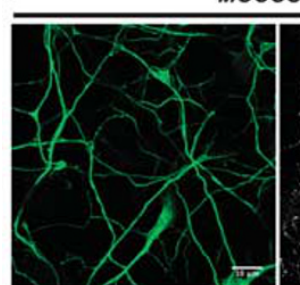

TUJ-1 $\operatorname{mocos}^{+/+}$

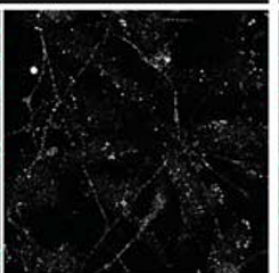

SYNAPSIN

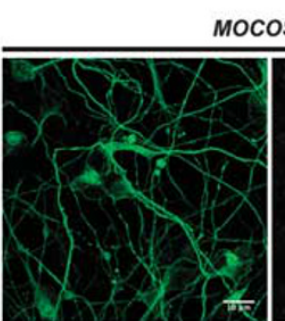

TUJ-1 $\operatorname{mocos}^{+1-}$

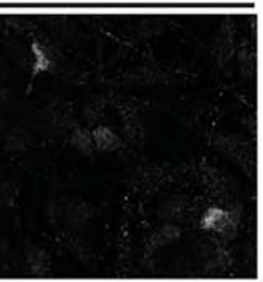

SYNAPSIN

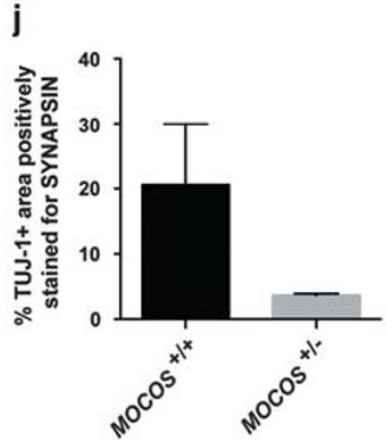

iPSCs displayed a lower number of SYNAPSIN+ synaptic buttons (Figures $4 i$ and $j$ ). These results confirm that MOCOS is a newly identified molecule having a role during neurodevelopment and neurotransmission.

\section{DISCUSSION}

Together, our results highlight MOCOS as a new candidate molecule implicated in ASD physiopathogeny and unveil novel biological functions associated with MOCOS, including roles in 
Figure 4. MOCOS expression alterations result in neurotransmission and synaptic defects. (a) Paralyzing effects of aldicarb (a cholinesterase inhibitor) in wild-type (N2) worms and mocs-1 mutants (tm3708, ok3439). A strain resistant to aldicarb, ric-3, was used as an experimental control for the aldicarb assay. Note that mocs-1 mutants exhibit a resistance to the paralytic effect of aldicarb ( $n=50-70$ animals per group). (b and $\mathbf{c}$ ) Representative pictures (b) and quantitative analysis of the mean fluorescence intensity (c) of the YFP-tagged neuropeptide NLP-21 in the dorsal cord axons of cholinergic motor neurons for each of the indicated C. elegans strains. (d) Representative pictures showing the NLP-21 loading in neuronal soma (upper row) and coelomocytes (lower row) for each of the indicated strains. (e) Quantitative analysis of the NLP-21 loading in neuronal soma (upper panel) and coelomocytes (lower panel), as measured by mean fluorescent intensity relative to N2 animals. (f) Bar charts depicting the mean area of NLP-21-loaded dense core vesicles present in neuronal soma (upper panel) and coelomocytes (lower panel), in mocs-1 mutants relative to wild-type N2 animals. (g) Schematic representation of the TALEN-based strategy used to monoallelically invalidate MOCOS in human iPSCs. Half arrows indicate primer sites for PCR (P1, P2, P3 and P4). Red triangles indicate the FLPo recognition target site. (h) iPSC-derived neural stem cells were differentiated into neurons and immunostained with anti-TUJ1 and anti-SYNAPSIN antibodies. Nuclei were counterstained with DAPI. (i and j) Representative pictures (i) and quantification (j) of synaptic density in $M O C O S^{+/-}$ neural stem cells-derived neurons compared with those derived from isogenic control lines $\left(\mathrm{MOCOS}^{+/+}\right)$. Data are represented as mean \pm s.d. ${ }^{*} P<0.05$. Scale bars: $100 \mu \mathrm{m}$ (h, left panel); $10 \mu \mathrm{m}$ (b and $\left.\mathbf{i}\right) ; 4 \mu \mathrm{m}$ (h, right panels) and $2 \mu \mathrm{m}$ (d).

stress response and neurotransmission. Bearing in mind that MOCOS was found downregulated in most ASD patients of our cohort, the phenotypes observed in our cellular and animal models are of the most relevance to establish a causal link between MOCOS misregulation and ASD-associated clinical symptoms. Of interest, metabolic defects have been associated with autistic symptoms with a prevalence higher than that found in the general population, ${ }^{33}$ including sulfur metabolism ${ }^{38}$ and purine metabolism disorders. ${ }^{32}$ Moreover, recent studies have highlighted that antipurinergic therapy is efficient in reducing autism-like features in animal models of ASD. ${ }^{39-41}$ In addition, xanthines and more generally purine derivatives were also found to have psychostimulant properties through their possible interaction with multiple receptors. ${ }^{42}$ However, this study represents the first report describing MOCOS as a putative modulator of other developmental and adult functions.

Among body metabolism biomarkers in ASD, and as shown in our results, oxidative-stress indicators stand out. It has been observed that urinary antioxidants are found in smaller amounts in ASD patients and their levels of production are correlated with symptom severity. ${ }^{43}$ Misexpressed molecules include, for example, glutathione, advanced glycation end products, superoxide dismutase and metal-binding proteins such as transferrin (iron) and ceruloplasmin (copper). ${ }^{44}$ Lately, a diminished seric expression of glutathione, glutathione peroxidase, methionine and cysteine has been highlighted in a meta-analysis from 29 studies on ASD subjects. ${ }^{45}$ Along this line, purines and purine-associated enzymes are recognized markers of oxidative stress. ROS are generated during the production of uric acid, catalyzed by xanthine oxidase and $\mathrm{XDH}^{46}$ Conversely, uric acid is nowadays recognized as a protective factor acting as a ROS scavenger. ${ }^{47,48}$ Interestingly, allopurinol, a xanthine oxidase inhibitor, was found efficient in reducing symptoms, especially epileptic seizures, in ASD patients displaying high levels of uric acid. ${ }^{49}$ However, in our cohort, only 3 out of 10 patients exhibited an abnormal uric acid secretion. It can therefore be postulated that still unknown other MOCOSassociated mechanisms may have a role in the unbalanced stress response observed in ASD OSCs.

According to our results, MOCOS appears to be also associated with neurotransmission anomalies that have previously been proved to be strongly affected in ASD. ${ }^{1}$ It has not escaped our notice that the enzymatic nature of our candidate molecule fits well with the wide ranging and unspecific neurotransmitter imbalance observed in ASD. As a molecule involved in the formation of dense core vesicles and, further down, neurotransmitter secretion, MOCOS seems to act on the container rather than the content, on the vehicle rather than one of the transported components. Interestingly, other studies have highlighted the potential role of dense core vesicles-associated proteins in ASD. ${ }^{50-52}$

In addition, as a key element of purine metabolic pathway, MOCOS may indirectly be involved in an extensive stimulation of neurotransmitter machinery. Indeed, it is established that xanthines and more generally purine derivatives display psychostimulant properties, acting not only at the $A 1$ and $A 2 A$ subtypes of the adenosine receptors ${ }^{53}$ but also other central receptors as $\beta 1 / \beta 2$ Adrenergic, 5-HT1 serotonin, 5-HT2 serotonin, muscarinic, nicotinic, GABA A, $\mathrm{Ca}^{2+}$ channel, in the cortex and the cerebellum. ${ }^{42}$

Identifying and manipulating downstream effectors of MOCOS will be the next critical step to better understand its mechanisms of action. In parallel, we plan to ascertain some of its upstream regulators. For example, bioinformatic analyses revealed that the promoter region of MOCOS includes conserved binding sites for transcription factors such as GATA3 and NRF2. In addition, other putative interactors, such as the NAD-dependent deacetylase sirtuin-1 (SIRT1), may have a regulatory role on MOCOS expression. Interestingly, these three genes have been associated with ASD, fragile $X$ syndrome, epilepsy and/or oxidative stress. ${ }^{54-57}$ In conclusion, our study opens an unexplored new avenue for the study of MOCOS in ASD, and could set bases for the development of new diagnostic tools as well as the search of new therapeutics.

\section{CONFLICT OF INTEREST}

The authors declare no conflict of interest.

\section{ACKNOWLEDGMENTS}

We thank Dr Mani Sahebjam (Edouard Toulouse Hospital, Marseille), Dr Claude Guinard (Valvert Hospital, Marseille) and Dr Rémy Defer (Montperrin Hospital, Aix-enProvence) for their help in recruiting the participants in this study. We are also thankful to Claudine Rumeau for magnetic resonance imaging analyses, Kevin Baranger for providing neuronal culture as well as Stéphanie Frecourt, Rim Yamani and Rupa Devi Soligalla for technical assistance. We thank the C. elegans Gene Knockout Consortium, S Mitani and the National Bioresource Project and J Kaplan and L Bianchi for generously providing strains. Other strains used in this study were provided by the Caenorhabditis Genetics Center, which is funded by NIH Office of Research Infrastructure Programs (P40 OD010440). We also thank the C. elegans TransgenOme Project and Knudra. This research was funded by Fondation de France (\#2004 010855; \#2004 010825; \#2007 005780), CNRS and Aix-Marseille University.

\section{REFERENCES}

1 Gepner B, Feron F. Autism: a world changing too fast for a mis-wired brain? Neurosci Biobehav Rev 2009; 33: 1227-1242.

2 Levy SE, Mandell DS, Schultz RT. Autism. Lancet 2009; 374: 1627-1638.

3 Lai MC, Lombardo MV, Baron-Cohen S. Autism. Lancet 2014; 383: 896-910.

4 Weintraub K. The prevalence puzzle: autism counts. Nature 2011; 479: 22-24.

5 Rubenstein JL. Three hypotheses for developmental defects that may underlie some forms of autism spectrum disorder. Curr Opin Neurol 2010; 23: 118-123.

6 Travers BG, Adluru N, Ennis C, Tromp do PM, Destiche D, Doran S et al. Diffusion tensor imaging in autism spectrum disorder: a review. Autism Res 2012; 5: 289-313.

7 Kern JK, Jones AM. Evidence of toxicity, oxidative stress, and neuronal insult in autism. J Toxicol Environ Health B Crit Rev 2006; 9: 485-499.

8 Zeidan-Chulia F, Salmina AB, Malinovskaya NA, Noda M, Verkhratsky A, Moreira JC. The glial perspective of autism spectrum disorders. Neurosci Biobehav Rev 2014; 38: $160-172$. 
9 Geschwind DH. Genetics of autism spectrum disorders. Trends Cogn Sci 2011; 15: 409-416.

10 Berg JM, Geschwind DH. Autism genetics: searching for specificity and convergence. Genome Biol 2012; 13: 247.

11 Abrahams BS, Geschwind DH. Advances in autism genetics: on the threshold of a new neurobiology. Nat Rev Genet 2008; 9: 341-355.

12 Walsh $\mathrm{P}$, Elsabbagh $\mathrm{M}$, Bolton $\mathrm{P}$, Singh I. In search of biomarkers for autism: scientific, social and ethical challenges. Nat Rev Neurosci 2011; 12: 603-612.

13 Murrell W, Feron F, Wetzig A, Cameron N, Splatt K, Bellette B et al. Multipotent stem cells from adult olfactory mucosa. Dev Dyn 2005; 233: 496-515.

14 Delorme B, Nivet E, Gaillard J, Haupl T, Ringe J, Deveze A et al. The human nose harbors a niche of olfactory ectomesenchymal stem cells displaying neurogenic and osteogenic properties. Stem Cells Dev 2010; 19: 853-866.

15 Boone N, Loriod B, Bergon A, Sbai O, Formisano-Treziny C, Gabert J et al. Olfactory stem cells, a new cellular model for studying molecular mechanisms underlying familial dysautonomia. PLoS One 2010; 5: e15590.

16 Matigian N, Abrahamsen G, Sutharsan R, Cook AL, Vitale AM, Nouwens A et al. Disease-specific, neurosphere-derived cells as models for brain disorders. Dis Model Mech 2010; 3: 785-798.

17 Mendel RR, Kruse T. Cell biology of molybdenum in plants and humans. Biochim Biophys Acta 2012; 1823: 1568-1579.

18 Ichida K, Matsumura T, Sakuma R, Hosoya T, Nishino T. Mutation of human molybdenum cofactor sulfurase gene is responsible for classical xanthinuria type II. Biochem Biophys Res Commun 2001; 282: 1194-1200.

19 Peretz H, Naamati MS, Levartovsky D, Lagziel A, Shani E, Horn I et al. Identification and characterization of the first mutation (Arg776Cys) in the C-terminal domain of the Human Molybdenum Cofactor Sulfurase (HMCS) associated with type II classical xanthinuria. Mol Genet Metab 2007; 91: 23-29.

20 Yamamoto T, Moriwaki Y, Takahashi S, Tsutsumi Z, Tuneyoshi K, Matsui K et al. Identification of a new point mutation in the human molybdenum cofactor sulferase gene that is responsible for xanthinuria type II. Metabolism 2003; 52: 1501-1504.

21 Girard SD, Deveze A, Nivet E, Gepner B, Roman FS, Feron F. Isolating nasal olfactory stem cells from rodents or humans. J Vis Exp 2011; 54: e2762; doi:10.3791/2762.

22 Sieburth D, Madison JM, Kaplan JM. PKC-1 regulates secretion of neuropeptides. Nat Neurosci 2007; 10: 49-57.

23 Sarov M, Murray Jl, Schanze K, Pozniakovski A, Niu W, Angermann K et al. A genome-scale resource for in vivo tag-based protein function exploration in $\mathrm{C}$. elegans. Cell 2012; 150: 855-866.

24 Kamath RS, Martinez-Campos M, Zipperlen P, Fraser AG, Ahringer J. Effectiveness of specific RNA-mediated interference through ingested double-stranded RNA in Caenorhabditis elegans. Genome Biol 2001; 2: RESEARCH0002.

25 Mahoney TR, Luo S, Nonet ML. Analysis of synaptic transmission in Caenorhabditis elegans using an aldicarb-sensitivity assay. Nat Protoc 2006; 1: 1772-1777.

26 Liu GH, Qu J, Suzuki K, Nivet E, Li M, Montserrat N et al. Progressive degeneration of human neural stem cells caused by pathogenic LRRK2. Nature 2012; 491: 603-607.

27 Parker KJ, Garner JP, Libove RA, Hyde SA, Hornbeak KB, Carson DS et al. Plasma oxytocin concentrations and OXTR polymorphisms predict social impairments in children with and without autism spectrum disorder. Proc Natl Acad Sci USA 2014; 111: 12258-12263.

28 Chez MG, Dowling T, Patel PB, Khanna P, Kominsky M. Elevation of tumor necrosis factor-alpha in cerebrospinal fluid of autistic children. Pediatr Neurol 2007; 36: 361-365.

29 Depino AM. Peripheral and central inflammation in autism spectrum disorders. Mol Cell Neurosci 2013; 53: 69-76.

30 Ashwood P, Enstrom A, Krakowiak P, Hertz-Picciotto I, Hansen RL, Croen LA et al. Decreased transforming growth factor beta1 in autism: a potential link between immune dysregulation and impairment in clinical behavioral outcomes. J Neuroimmunol 2008; 204: 149-153.

31 Kalkman HO. A review of the evidence for the canonical Wnt pathway in autism spectrum disorders. Mol Autism 2012; 3: 10.

32 Page T, Coleman M. Purine metabolism abnormalities in a hyperuricosuric subclass of autism. Biochim Biophys Acta 2000; 1500: 291-296.

33 Manzi B, Loizzo AL, Giana G, Curatolo P. Autism and metabolic diseases. J Child Neurol 2008; 23: 307-314.

34 McGinnis WR. Oxidative stress in autism. Altern Ther Health Med 2004; 10: 22-36.

35 James SJ, Melnyk S, Jernigan S, Cleves MA, Halsted CH, Wong DH et al. Metabolic endophenotype and related genotypes are associated with oxidative stress in children with autism. Am J Med Genet B Neuropsychiatr Genet 2006; 141B: 947-956.

36 Garattini E, Fratelli M, Terao M. Mammalian aldehyde oxidases: genetics, evolution and biochemistry. Cell Mol Life Sci 2008; 65: 1019-1048.

37 Sudhof TC. Neuroligins and neurexins link synaptic function to cognitive disease. Nature 2008; 455: 903-911.

38 Waring RH, Klovrza LV. Sulphur metabolism in autism. J Nutr Environ Med 2000; 10: $25-32$.

39 Naviaux JC, Schuchbauer MA, Li K, Wang L, Risbrough VB, Powell SB et al. Reversal of autism-like behaviors and metabolism in adult mice with single-dose antipurinergic therapy. Transl Psychiatry 2014; 4: e400.

40 Naviaux JC, Wang L, Li K, Bright AT, Alaynick WA, Williams KR et al. Antipurinergic therapy corrects the autism-like features in the Fragile $\mathrm{X}$ (Fmr1 knockout) mouse model. Mol Autism 2015; 6: 1.

41 Naviaux RK, Zolkipli Z, Wang L, Nakayama T, Naviaux JC, Le TP et al. Antipurinergic therapy corrects the autism-like features in the poly(IC) mouse model. PLoS One 2013; 8: e57380.

42 Shi D, Daly JW. Chronic effects of xanthines on levels of central receptors in mice. Cell Mol Neurobiol 1999; 19: 719-732.

43 Damodaran LP, Arumugam G. Urinary oxidative stress markers in children with autism. Redox Rep 2011; 16: 216-222.

44 Goldani AA, Downs SR, Widjaja F, Lawton B, Hendren RL. Biomarkers in autism. Front Psychiatry 2014; 5: 100.

45 Frustaci A, Neri M, Cesario A, Adams JB, Domenici E, Dalla Bernardina B et al. Oxidative stress-related biomarkers in autism: systematic review and meta-analyses. Free Radic Biol Med 2012; 52: 2128-2141.

46 Schulz E, Gori T, Munzel T. Oxidative stress and endothelial dysfunction in hypertension. Hypertension Res 2011; 34: 665-673.

47 Nabipour I, Sambrook PN, Blyth FM, Janu MR, Waite LM, Naganathan V et al. Serum uric acid is associated with bone health in older men: a cross-sectional population-based study. J Bone Miner Res 2011; 26: 955-964.

48 Waring WS, Webb DJ, Maxwell SR. Systemic uric acid administration increases serum antioxidant capacity in healthy volunteers. J Cardiovasc Pharmacol 2001; 38: 365-371.

49 Coleman M, Landgrebe M, Landgrebe A. Progressive seizures with hyperuricosuria reversed by allopurinol. Arch Neurol 1974; 31: 238-242.

50 Paemka L, Mahajan VB, Skeie JM, Sowers LP, Ehaideb SN, Gonzalez-Alegre P et al. PRICKLE1 interaction with SYNAPSIN I reveals a role in autism spectrum disorders. PLoS One 2013; 8: e80737.

51 Sadakata T, Shinoda Y, Sato A, Iguchi H, Ishii C, Matsuo M et al. Mouse models of mutations and variations in autism spectrum disorder-associated genes: mice expressing Caps2/Cadps2 copy number and alternative splicing variants. Int $J$ Environ Res Public Health 2013; 10: 6335-6353.

52 Zhao H, Kim Y, Park J, Park D, Lee SE, Chang I et al. SCAMP5 plays a critical role in synaptic vesicle endocytosis during high neuronal activity. J Neurosci 2014; 34: 10085-10095.

53 Lorist MM, Tops M. Caffeine, fatigue, and cognition. Brain Cogn 2003; 53: 82-94.

54 Furnari MA, Saw CL, Kong AN, Wagner GC. Altered behavioral development in Nrf2 knockout mice following early postnatal exposure to valproic acid. Brain Res Bull 2014; 109: 132-142.

55 Kumari D, Usdin K. Polycomb group complexes are recruited to reactivated FMR1 alleles in Fragile $X$ syndrome in response to FMR1 transcription. Hum Mol Genet 2014; 23: 6575-6583.

56 Napoli E, Wong S, Hertz-Picciotto I, Giulivi C. Deficits in bioenergetics and impaired immune response in granulocytes from children with autism. Pediatrics 2014; 133: e1405-e1410.

57 Rout UK, Clausen P. Common increase of GATA-3 level in PC-12 cells by three teratogens causing autism spectrum disorders. Neurosci Res 2009; 64: 162-169.

(c) This work is licensed under a Creative Commons Attributioncc. NonCommercial-NoDerivs 4.0 International License. The images or other third party material in this article are included in the article's Creative Commons license, unless indicated otherwise in the credit line; if the material is not included under the Creative Commons license, users will need to obtain permission from the license holder to reproduce the material. To view a copy of this license, visit http:// creativecommons.org/licenses/by-nc-nd/4.0/

(c) The Author(s) 2016

Supplementary Information accompanies the paper on the Molecular Psychiatry website (http://www.nature.com/mp) 